\title{
Material Concepts
}

\section{Dear Reader,}

Emission levels of less than $90 \mathrm{~g}$ of $\mathrm{CO}_{2}$ per $\mathrm{km}$ are hardly possible without electrification, alternative fuels or lightweight design. The latest Shell Passenger Car Scenarios forecast a fleet of 10.1 million electric vehicles by 2040 , while the number of petrol- or dieselpowered vehicles will fall to 30.7 million. McKinsey expects that only around $5 \%$ of vehicles will have an internal combustion engine in 2050. The $2011 \mathrm{EU}$ White Paper on transport policy predicts that the use of conventionally fuelled cars in urban transport will halve by 2030 .

There are therefore no unanimous forecasts for the number of electric cars in the future. Electric mobility is not predictable. Customers need to be convinced - both emotionally and economically. At the same time, the need for mobility is falling, and mobility is still strongly determined by emotions. So what are customers looking for and what price are they prepared to pay for an energy-optimised vehicle?

In addition to applying suitable measures to the powertrain, lightweight design is an effective means of reducing vehicle weight and emissions. In automotive engineering, there is huge potential not only in the vehicle body but also in loadbearing or safety-relevant components, especially if composite materials are used. The key point, however, is the cost-effectiveness of lightweight design concepts, particularly when we need to consider not only the additional costs of lightweight construction of up to 5 Euros/kg but also the added cost of an electrified powertrain, as well as the fact that the use of different materials will mean that repairs might become more complex and therefore more expensive.

These questions were reason enough to make the subject of lightweight design the leading topic of this issue of ATZ.
Recent innovations in components and materials are evidence of significant progress in aspects of energy and weight efficiency, safety, functionality, design and comfort. This topic is an ideal complement to the latest issue of our magazine lightweight design (lwd), which, in the first edition of 2015, turns its attention to chassis components, and shows how ZF combines material substitution and lightweight design in a wheel-guiding transverse spring made of GRP.

Choosing the right material at the right place is an ongoing challenge for developers and design engineers. It involves enabling new material concepts to be put into production from an economic perspective, while at the same time also addressing life cycle issues. The focus remains on the efficient use of energy and therefore the sustainable protection of resources. In modern automotive engineering, the entire process chain must remain visible at all times.

Best regards,

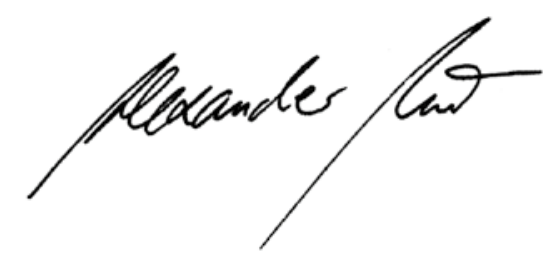

Dr. Alexander Heintzel, Editor in Chief Wiesbaden, 22 January 2015

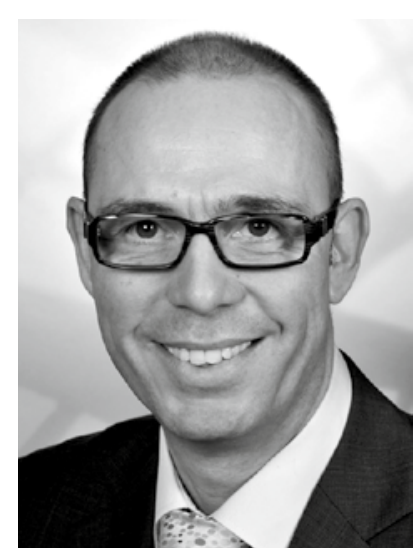

